\title{
Meshing effects of the 3-D FEM numerical modeling in seismo-electromagnetics: An application in selectivity of seismic electric signal (SES)*
}

\author{
Qiao Wang Guangjing Xu Zhanhui Li and Qinghua Huang* \\ Department of Geophysics, School of Earth and Space Sciences, Peking University, Beijing 100871, China
}

\begin{abstract}
We investigated how density and quality of mesh around interest domain affect electromagnetic (EM) responses of 3D Earth layered media using finite element method (FEM). Effect of different mesh shapes was also investigated using a method of mixing structured and unstructured mesh. As a case study, we estimated the effects of meshing on selectivity phenomenon of seismic electric signal (SES). Our results suggest that the relative errors resulting from mesh effects may not be negligible, which may lead to some unconvincing explanation of the SES selectivity based on the numerical modeling results.
\end{abstract}

Key words: 3D electromagnetic numerical modeling; FEM; meshing; SES

CLC number: P315.72+1 Document code: A

\section{Introduction}

As a new branch of geoelectromagnetism, seismoelectromagnetics focuses on the electromagnetic phenomena associated with earthquake, and aims at understanding the nature of seismogenic process from the viewpoint of electromagnetism (Huang, 2004). There are numerous independent reports on the electromagnetic (EM) phenomena associated with earthquakes (Central Earthquake Working Committee, 1969; Sobolev, 1975; Zhao and Qian, 1978; Varotsos and Alexopoulos, 1984a, b; Qian et al., 1985; Fraser-Smith et al., 1990; Johnston, 1997; Hayakawa et al., 2000; Nagao et al., 2002; Hattori et al., 2004; Huang and Liu, 2006). Such EM phenomena, especially ultra-low-frequency (ULF) EM signals, have ever been attempted to being applied to short-term prediction of earthquakes (Varotsos, 2005; Hayakawa et al., 2008). Although the seismoEM phenomena have not been fully understood till now, there are some continuous attempts to the study on possible mechanisms of seismo-EM phenomena (Bernard,

\footnotetext{
* Received 19 July 2011; accepted in revised form 6 September 2011; published 10 December 2011.

† Corresponding author. e-mail: huangq@pku.edu.cn

(c) The Seismological Society of China and Springer-Verlag Berlin Heidelberg 2011
}

1992; Johnston, 1997; Vallianatos and Tzanis, 1998; Guo et al., 1999; Huang, 2002). Much attention has been paid to some debated phenomena such as the selectivity phenomenon of seismic electric signal (SES) (Varotsos and Lazaridou, 1991). Besides theoretical model analysis (Varotsos et al., 1998; Varotsos, 2005), some laboratory analogue experiments (Meng and Dosso, 1990; Huang and Ikeya, 1998, 1999; Huang, 2005; Lin, 2010) and numerical modeling (Sarlis et al., 1999; Kulchitsky et al., 2004; Huang and Lin, 2010a, b) have been adopted in the explanation of the SES selectivity phenomenon.

Due to low cost and convenience for field tests and indoor experiments, numerical modeling becomes a useful tool in solving geo-EM problems, especially EM sounding. Recently, there are also some attempts to applying numerical technique to seismo-EM problems, e.g., Sarlis et al. (1999) made a numerical modeling with a conductive channel to explain the SES selectivity using the EM1DSH software, which was developed for calculating either the layered Earth model including displacement currents or the two-layer model with embedded thin sheets but without displacement currents (Hoversten and Becker, 1995). As a further study, Huang and Lin (2010a, b) made a numerical simulation 
on SES selectivity based on 3D finite element method (FEM, Xu, 1994). Their results indicated that either the heterogeneous ground surface or the underground conductive channel could lead to the selectivity phenomenon of SES.

In 3D EM numerical modeling, there are mainly three methods: finite-difference method (FDM), integral equation method (IEM) and FEM. In comparison with FDM and IEM, FEM is a flexible tool for complex models, because either triangular or tetrahedral elements it uses can deal with arbitrary geometry shapes (ore-bodies, cylindrical wells, topography, etc.) (Nam et al., 2007). Although many improvements have been achieved in FEM, such as use of nodal and edge elements (Ndlec, 1980, 1986; Sugeng, 1998; Jin et al., 1999; Yoshimura and Oshiman, 2002; Ma and Liu, 2006; Nam et al., 2007) for addressing the discontinuities of EM fields along material discontinuities, high accuracy solutions for modeling with complex geometry still requires much computational cost. Some researchers have investigated structured (rectangular, rectangle-triangle and hexahedral) and unstructured (triangular and tetrahedral) meshes for decreasing required memory and time in computation while increasing convergence and accuracy (Ren et al., 2008; Tang and Wang, 2008; Key and Weiss, 2006; Li and Key, 2007). Generally, unstructured mesh can improve accuracy of complex boundaries with the same nodes comparing with structured mesh, especially near some singular points (Tang and Wang, 2008). However, unstructured mesh also has its own deficiencies, e.g., the prior information about the domains under interest should be known in order to interact with the computer and the nodes distribution resulting from it is still not optimal. Consequently, the adaptive finite element method (AFEM) is developed recently in simulating the EM response of a petroleum engineering resistivity logging instrument in a borehole environment (Demkowicz and Vardapetyan, 1998; Rachowicz and Demkowicz, 2000, 2002; Cecot et al., 2003), which can automatically control the optimal distribution of the nodes to minimize the error of a prescribed quantity of interest with respect to the problem.

Huang and Lin (2010b) investigated the possible influences on 3D FEM numerical simulation of SES using a commercial FEM software, COMSOL Multiphysics ${ }^{\circledR}$. After unstructured meshing, they adopted different types of boundary condition, varied size of calculated region, various combinations of electrical parameters of the media, and different models containing heterogeneous ground surface in-homogeneity of conductivity or underground conductive channel. Their results indicated that selection of model parameters should be paid attetion to. SES selectivity may depend on the detailed electric structure and contrast of conductivity in the model.

In this study, we investigated the effects of meshing on the 3D numerical modeling of SES using the FEM software, COMSOL Multiphysics ${ }^{\circledR}$. After the validation test of the software, we investigated the effects of mesh quality, density and type based on various models with different meshing methods. As an example of modeling SES selectivity, we investigated numerically the response of SES to the meshing effects for a revised model after Sarlis et al. (1999) and Lin (2010).

\section{Methods}

We used the Maxwell's equations in the frequency domain (Zhdanov, 2009) under quasi-static approximation and the Dirichlet boundary condition (Abubakar et al., 2008) to deal with the boundary value problem. However, COMSOL does not solve Maxwell's equations directly. Instead it uses the Helmholtz potential decomposition with the Coulomb gauge condition to ensure that there are no spurious solutions in the EM potentials (Badea et al., 2001; Mitsuhata and Uchida, 2004). Following this approach we can get the weak form $(\mathrm{Xu}$, 1994) using Galerkin weighted residuals method. In this study, we only considered the case with low frequency in Earth media, so multi-grid solver (Mulder, 2006) was adopted in COMSOL, which has a high efficiency to solve the large system of linear-equations especially in $3 \mathrm{D}$.

In this study, we conducted quality measurement for elements. The quality measure is related to the aspect ratio, which means that anisotropic elements can get a low quality measure even though the element shape is reasonable (COMSOL, 2008). For tetrahedral elements, COMSOL Multiphysics ${ }^{\circledR}$ computes the mesh quality as

$$
q=\frac{72 \sqrt{3} V}{\left(\sum_{i=1}^{6} h_{i}^{2}\right)^{\frac{3}{2}}}
$$

where $V$ is the volume, and $h_{i}$ are the edge lengths of the tetrahedron. The values of $q$ would affect the numerical solutions significantly if $q$ is very small. The numerical results with a large $q$ will be more credible than those with a small one (COMSOL, 2008).

We judged whether a mesh is coarse or fine by es- 
timating the number of elements on unit length, $\lambda$, as

$$
\lambda=\frac{n}{L},
$$

where $n$ is the number of elements distributed on lines with a length of $L$. In this study, we specified some lines to increase or decrease $n$ according to our models.

\section{Validation test}

As a validation test of the COMSOL FEM software, we calculated the electric field $x$-component at $1 \mathrm{~Hz}$ on the top surface of a three-layer model with a point electrical dipole source (with $x$-directed dipole moment) in the third layer (Figure 1a). The point electric dipole source can be given in the model conveniently by the COMSOL FEM software. In this example, we adopted a point dipole source with a moment magnitude of $10^{4} \mathrm{~A} \cdot \mathrm{m}$ along the $x$-direction. We can obtain the theoretical solutions (Wei et al., 2007) for the same model. The numerical results obtained from COMSOL are in good consistency with the theoretical ones (Figure 1b). This can be further confirmed by the relative error given in Figure 1c.

Note that we conduct the following two element numbers in the validation test of FEM. The first is the ground line on the top of the first layer (Figure 1a) with $n_{1}=200$ and $L_{1}=20 \mathrm{~km}$. The second is the crossline on the horizontal plane of the point electric dipole source at a depth of $5 \mathrm{~km}$ (Figure 1a) with $n_{2}=500$ and $L_{2}=10 \mathrm{~km}$. Figure 2 shows the mesh quality on the horizontal plane of the point electric dipole source.
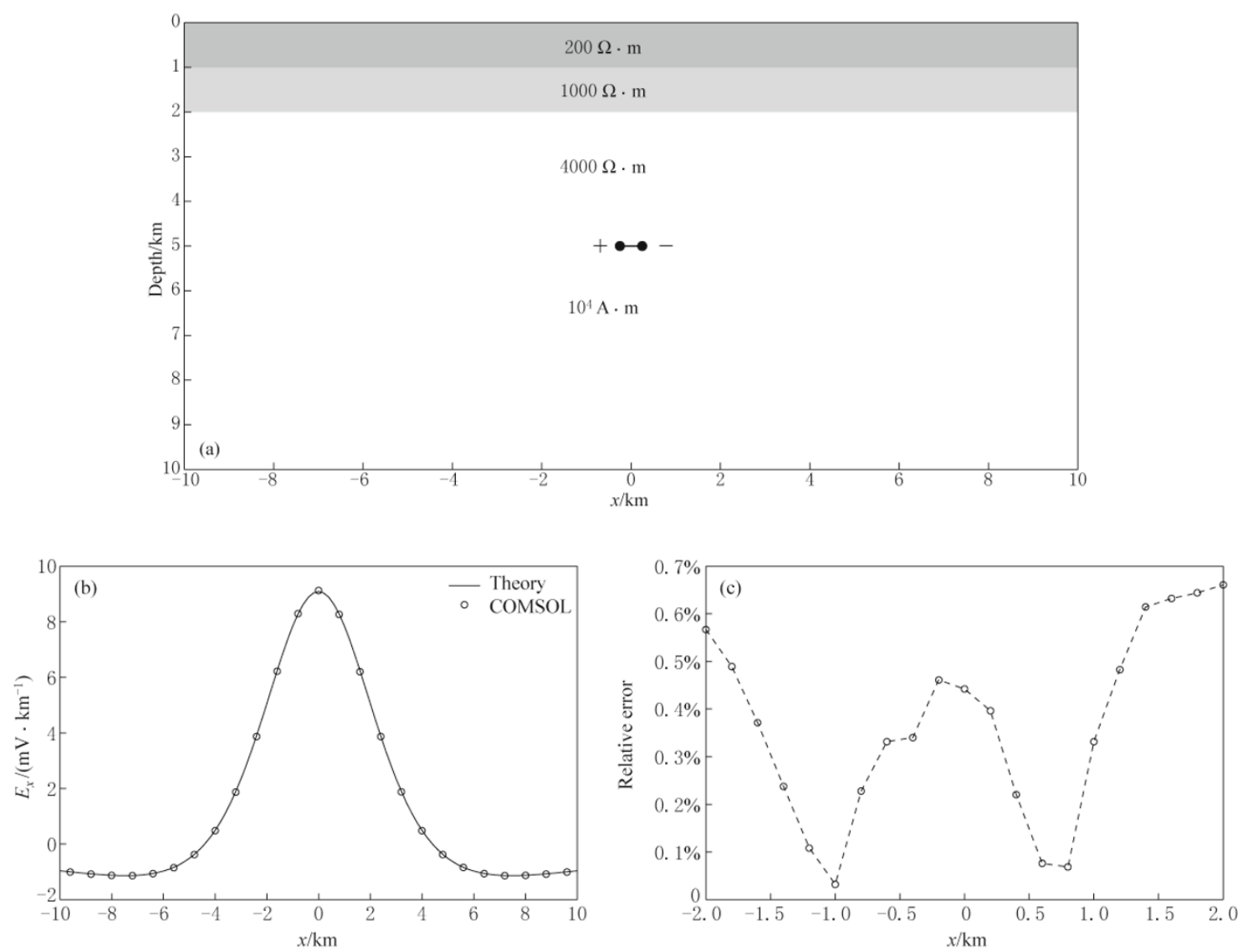

Figure 1 Validation test of the COMSOL FEM software. (a) An $x$-directed vertical cross-section passing through a point electric dipole source in a 3D three-layer model, which does not draw the air domain. The computation size is five times as much as the displaying model including air domains which has a conductivity of $10^{-14} \mathrm{~S} \cdot \mathrm{m}^{-1}$. (b) The $x$-components of electric field at $1 \mathrm{~Hz}$ are consistent well with the theoretical ones (black line). Circles represent the results of point electric dipole source. (c) The relative error between the numerical and theoretical results. 


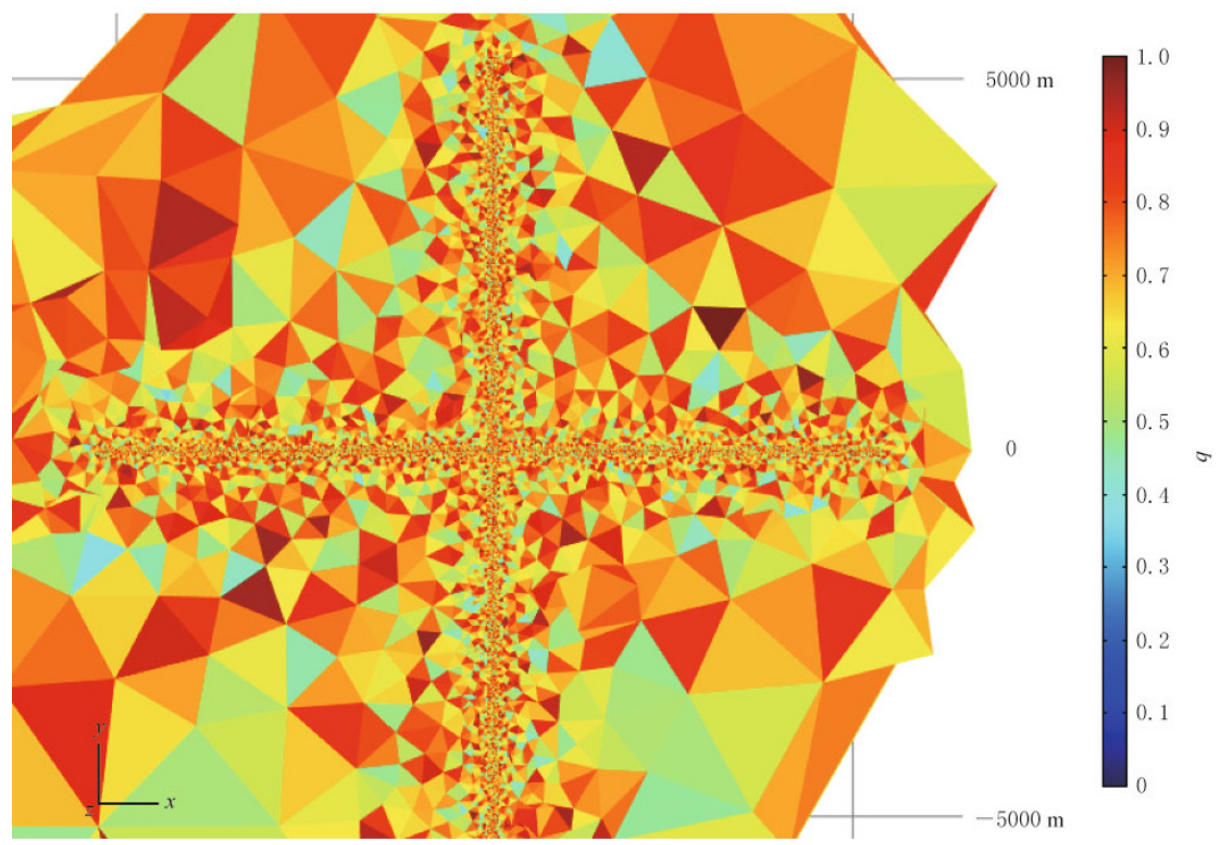

Figure 2 The mesh quality on the horizontal plane of the point electric dipole source with $n_{2}=500$ and $L_{2}=10 \mathrm{~km}$ (see text for details).

\section{Results}

\subsection{Mesh density effect}

Huang and Lin (2010b) studied the effects of the boundary condition and the size of calculated region comprehensively. In this study, we investigated the influence of the density of mesh around point source on numerical results.

As an example, Figure 3 a shows the numerical results obtained from the mesh conditions of $n_{1}=50$,
$L_{1}=20 \mathrm{~km}$, and $n_{2}=1, L_{2}=10 \mathrm{~km}$ for the model given in Figure 1a. It is coarser than that from the mesh conditions in Figure 1b. Figure 3a indicated that there are some clear deviations of the above numerical results from the theoretical results. Such phenomenon can be further confirmed by their relative errors (Figure 3b), which is at least one order larger than those obtained from the mesh conditions of $n_{1}=200, L_{1}=20 \mathrm{~km}$, and $n_{2}=500, L_{2}=10 \mathrm{~km}$ (Figure 1c).
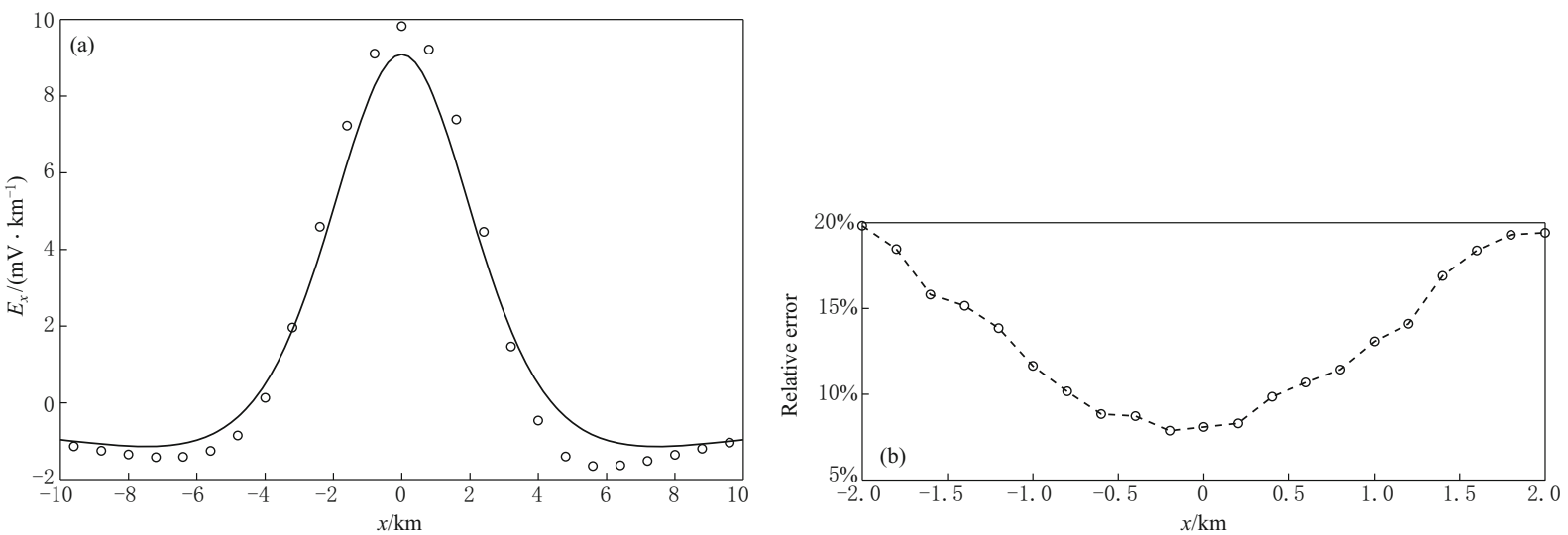

Figure 3 Results of electric field $x$-component at $1 \mathrm{~Hz}$ with different density of mesh. (a) The numerical results (circles) obtained from a relative coarse mesh around the point electric dipole source. The solid line represents the theoretical ones. (b) The relative error between the numerical and theoretical results. 


\subsection{Meshing way effect}

In order to investigate the possible effects due to the ways of meshing, we adopted different ways of meshing for the model given in Figure 1a and made the numerical calculations. Figure 4a shows the mesh quality of the meshing way with all the domains of the model using tetrahedral elements. The related numerical results are given in Figure 1b. As another meshing way, we first let the regions from top surface to the depth of point source, which are called "main domain", be tetrahedral elements, and let remaining regions, which are called "remained domain", be triangular prismatic
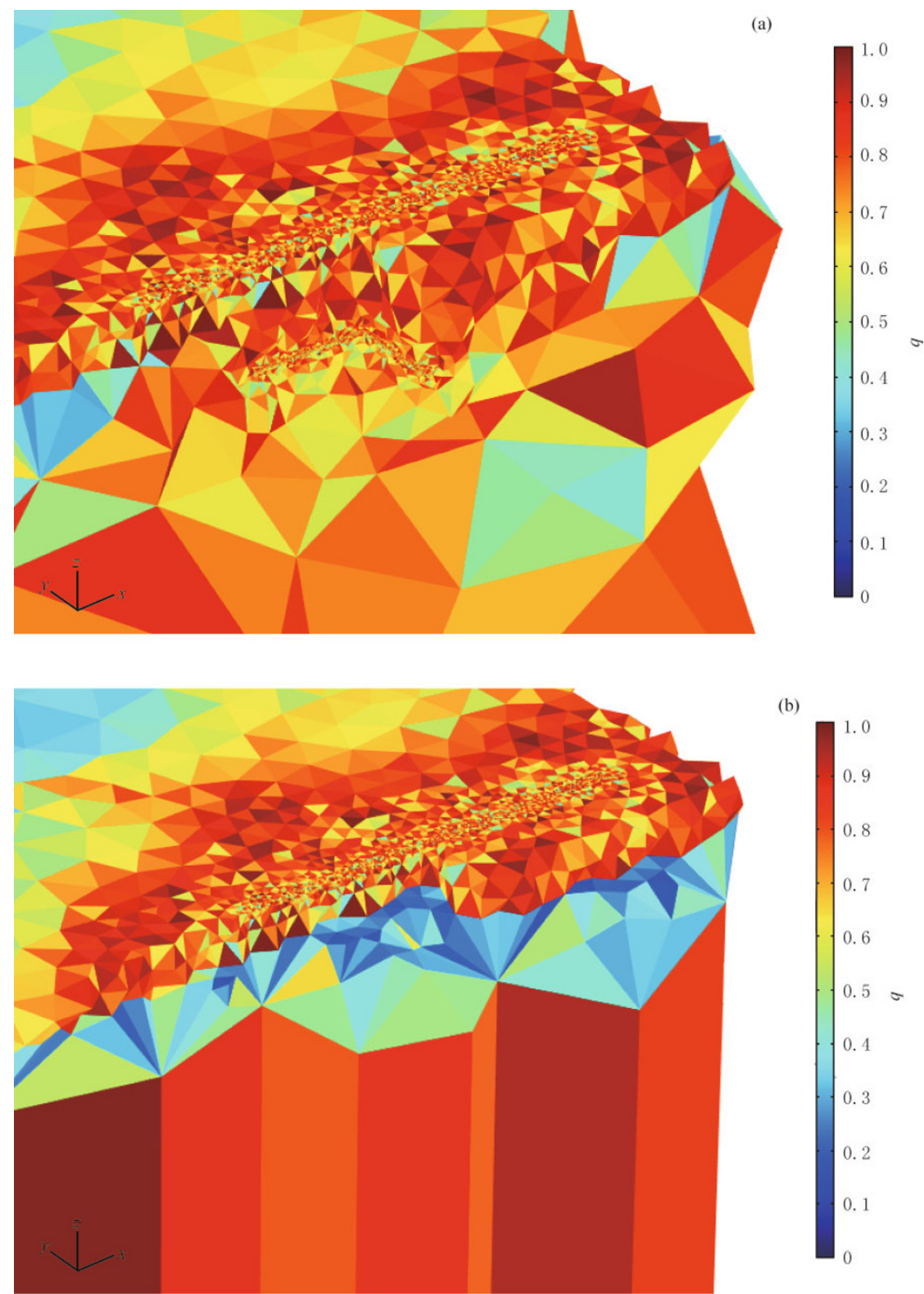

Figure 4 Mesh qualities for different ways of meshing. (a) The quality of mesh using tetrahedral elements for all domains. The average mesh quality is about 0.703 , with a maximum and a minimum of 0.958 and 0.174 , respectively. (b) The quality of mesh using tetrahedral elements and triangular prisms, "swept meshing". The average $q$ is about 0.498 , with a maximum and a minimum of 0.996 and 0.006 . 
elements by sweeping.

\subsubsection{Mixture meshing}

The average mesh quality, $q$, for the meshing way in Figure 4a, is about 0.703, with a maximum and a minimum of 0.958 and 0.174 , respectively. In the case of the mixture meshing given in Figure $4 \mathrm{~b}$, the average $q$ is about 0.498 , with a maximum and a minimum of 0.996 and 0.006 .

Figure 5a indicated that the numerical results of mixing elements of tetrahedron and prism have large deviations from those of the theoretical ones and tetra- hedral elements. The relative error between the numerical results of mixture meshing and the theoretical ones is more than 10\% (Figure 5b). Furthermore, its calculating time is about three times as much as that of the tetrahedral elements (see Table 1). Table 1 also gives the comparisons of some other solution parameters for these two ways of meshing method. Actually, prismatic elements are comparatively, simpler to implement, but the tetrahedral approach is potentially much more flexible, allowing high resolution where warranted, and hence more efficient 3D modeling (Sarakorn, et al., 2008).
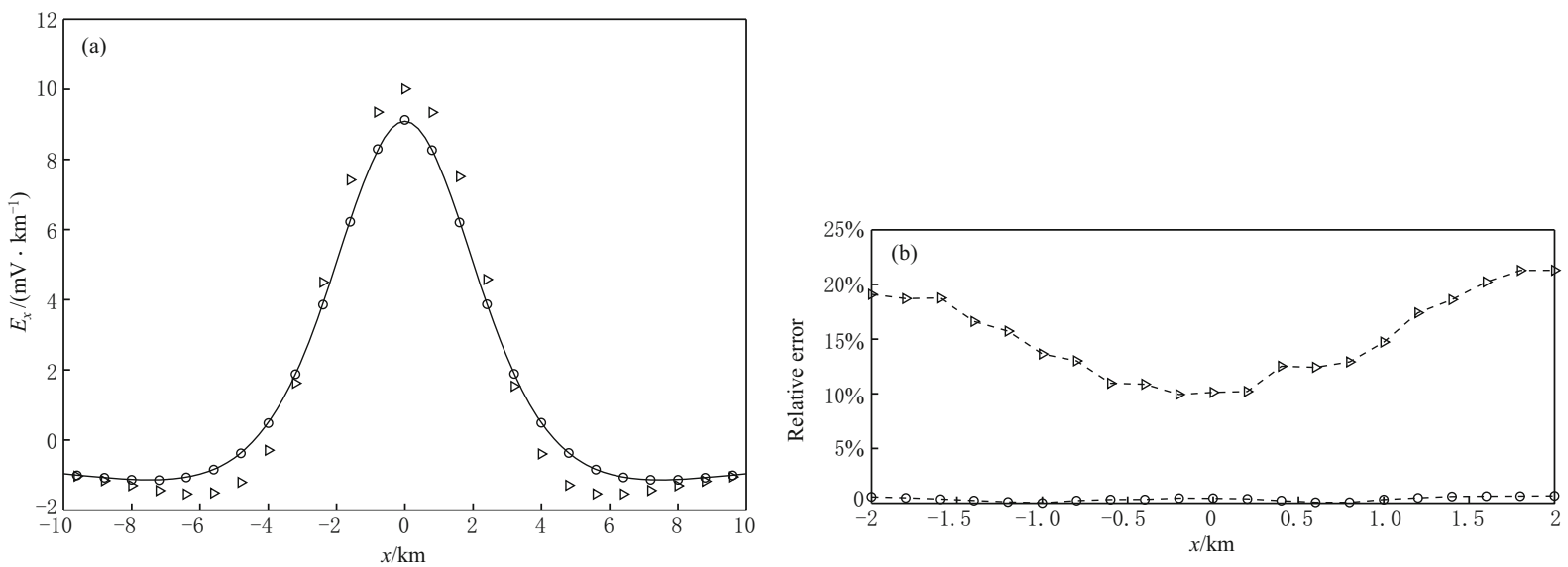

Figure 5 The $x$-component of electric field at $1 \mathrm{~Hz}$ for the mesh of Figure 4. (a) Comparison between the numerical results (circles and triangles) and the theoretical ones (black line). Circles are the same as results in the Figure 1b, and triangles represent the results from the mesh in Figure 4b. (b) Relative errors. The triangles (circles) represent the relative errors between numerical results and theoretical ones.

Table 1 Comparison of solution parameters between the different ways of meshing, tetrahedral and mixed elements

\begin{tabular}{cccccc}
\hline Meshing method & $\begin{array}{c}\text { Number of } \\
\text { elements }\end{array}$ & $\begin{array}{c}\text { Number of freedom } \\
\text { degrees }\end{array}$ & $\begin{array}{c}\text { Elapsed solution } \\
\text { time/s }\end{array}$ & $\begin{array}{c}\text { Number of iteration } \\
\text { for } 1 \mathrm{~Hz}\end{array}$ & $\begin{array}{c}\text { Number of iteration } \\
\text { for } 10 \mathrm{~Hz}\end{array}$ \\
\hline Tetrahedral elements & 294869 & 1873976 & 419.3 & 3 & 7 \\
Mixed elements & 219610 & 2052578 & 1283.8 & 31 & 81 \\
\hline
\end{tabular}

\subsubsection{Model with semi-torus using tetrahedral elements}

We added a semi-torus into the three-layer model. Figure $6 \mathrm{a}$ is its cross section, and Figure $6 \mathrm{~b}$ is its mesh quality under the conditions with $n_{1}=200$ and $L_{1}=20$ $\mathrm{km}, n_{2}=500$ and $L_{2}=10 \mathrm{~km}$, and $n_{3}=200$ and $L_{3}=3.77$ $\mathrm{km} . L_{3}$ is an arc line along the top of the semi-torus. Its average $q$ is about 0.794 with a maximum and a minimum of 0.977 and 0.385 . In the case of a relative coarse meshing given in Figure 6c, we let $n_{1}=50, n_{2}=2, n_{3}=2$, and let $L_{1}, L_{2}$, and $L_{3}$, respectively be the same as the above ones given in Figure 6b. It has a relative small- er average $q$ of 0.539 with a maximum and a minimum of 0.821 and 0.368 . Figure $7 \mathrm{a}$ indicated that some significant deviations occur between the numerical results from the meshes of Figures $6 \mathrm{~b}$ and $6 \mathrm{c}$. The maximum relative error is more than $10 \%$ near the center of semitorus (see Figure 7b).

\section{Discussion and conclusions}

As a case study, we adopted the model (see Figure 8) containing a relative conductive semi-torus channel and an ellipsoid underlying, which is revised from Sarlis 


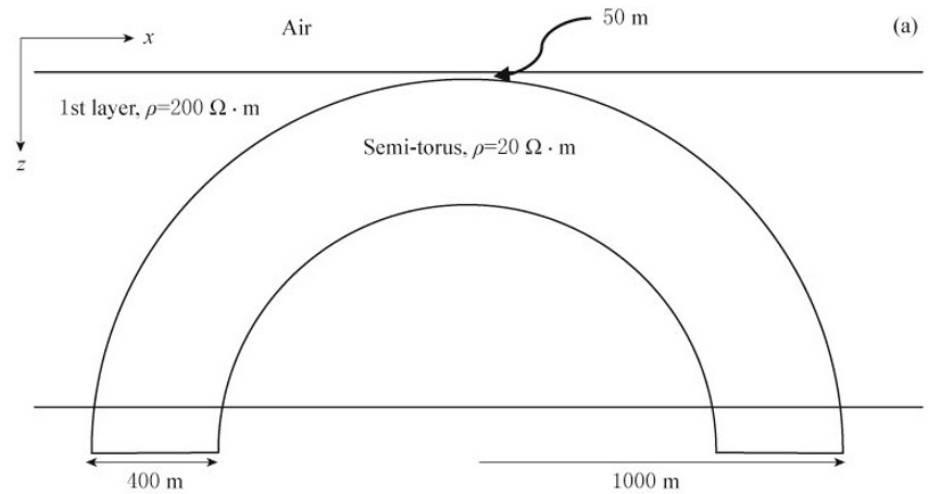

2nd layer, $\rho=1000 \Omega \cdot \mathrm{m}$

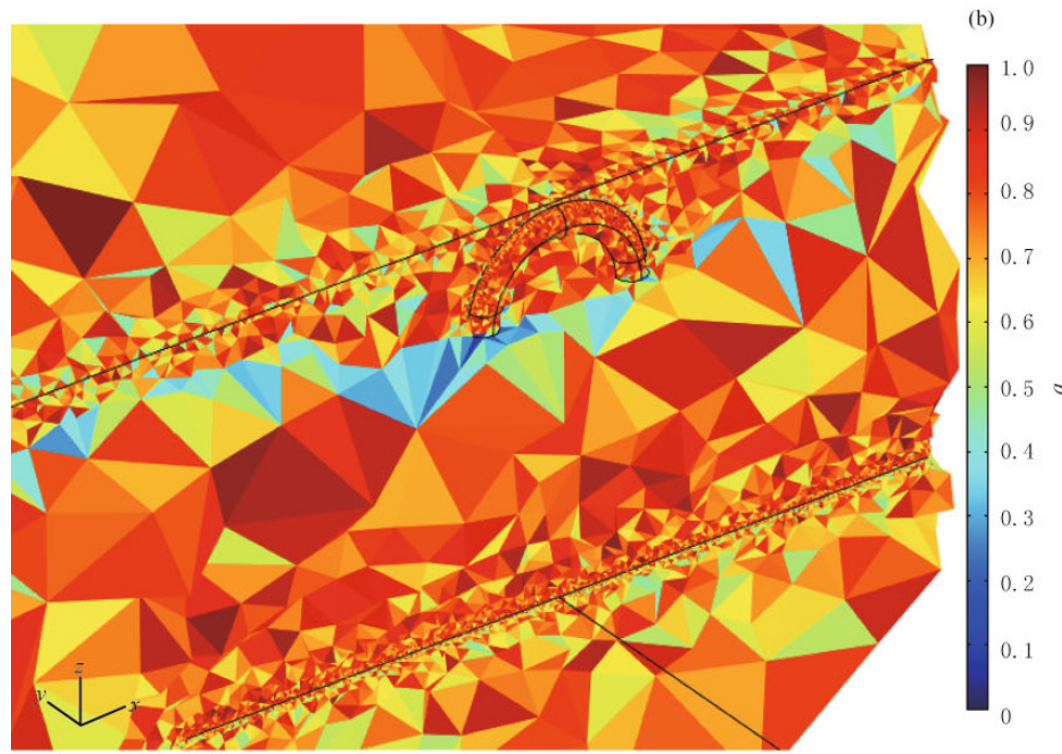

(c)

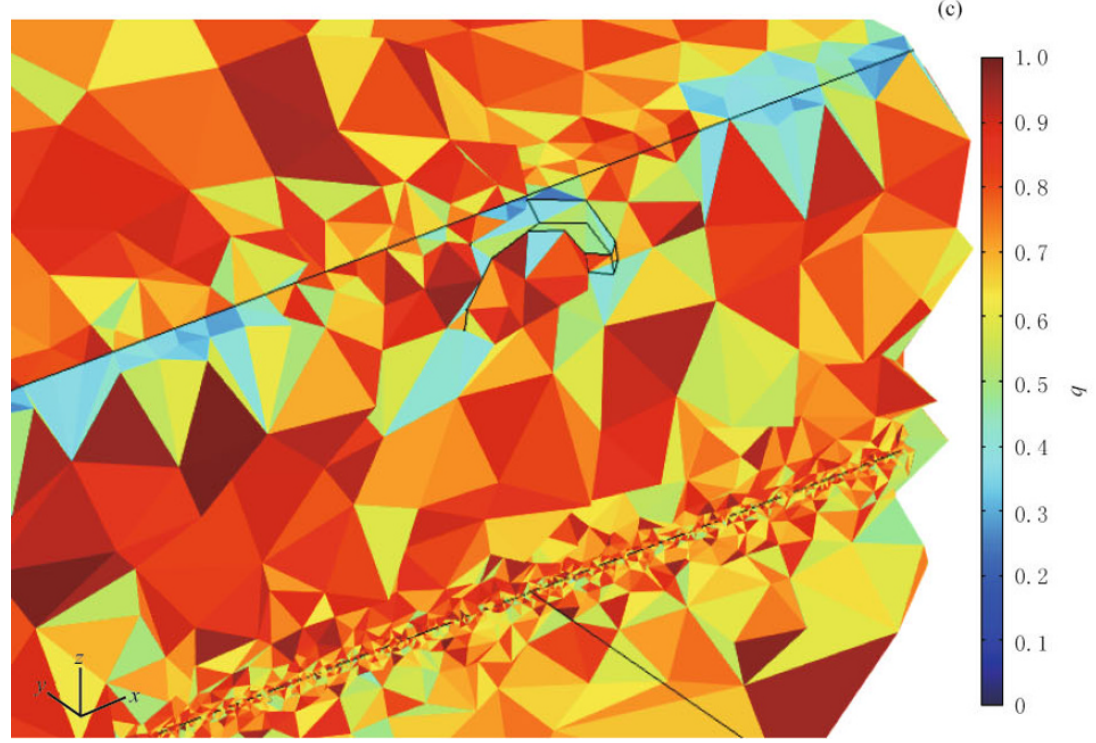

Figure 6 Cross sections of the semi-torus model with different mesh density. (a) Cross section for the resistivity structure of the semi-torus model. (b) A relative fine mesh. (c) A relative coarse mesh. 

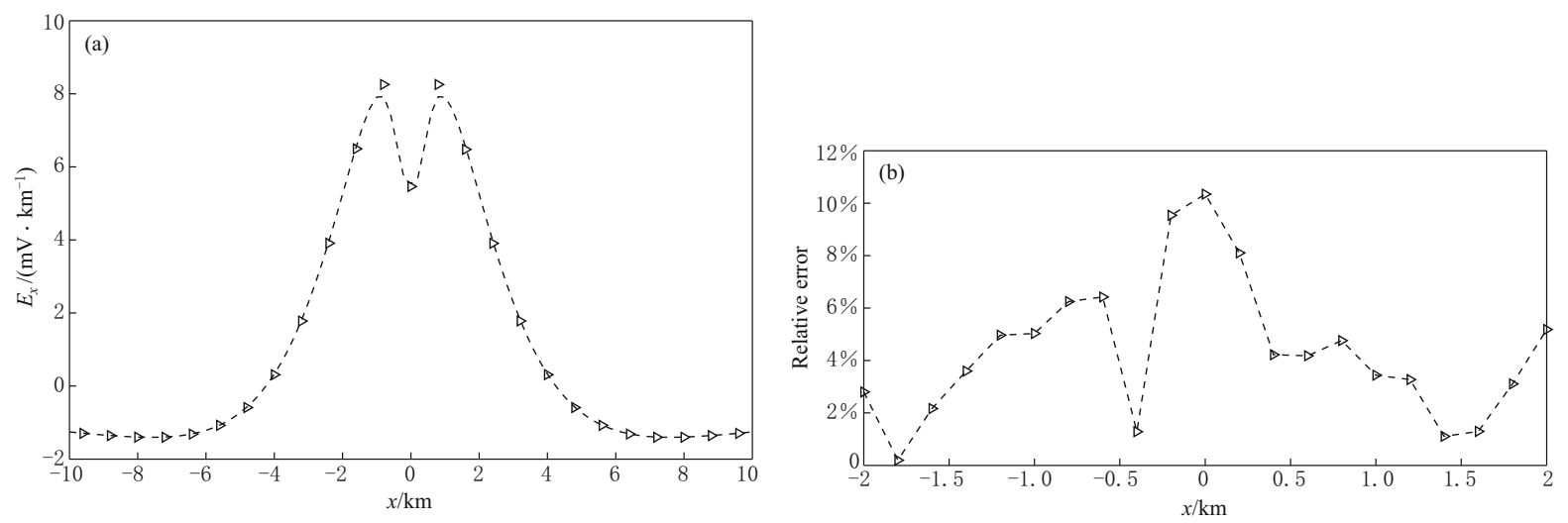

Figure 7 The $x$-component of electric field at $1 \mathrm{~Hz}$ with different mesh densities for the model in Figure 6a. (a) Dashed line represents the results of the relative fine mesh in Figure 6b. Triangles represent the results of relative coarse mesh in Figure 6c. (b) The relative error between the results of meshing conditions in Figure $6 \mathrm{~b}$ and Figure $6 \mathrm{c}$.

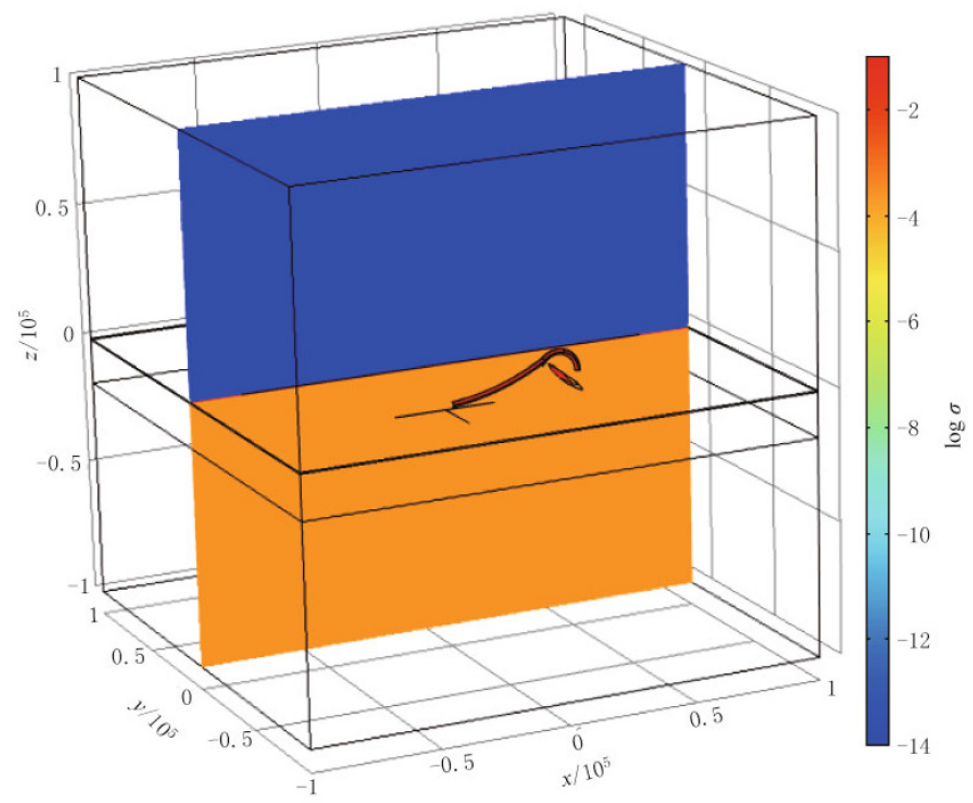

Figure 8 The 3D model with a conductive semi-torus channel and an ellipsoid modified from Sarlis et al. (1999) and Lin (2010). The color bar is logarithm scale for conductivity of the model. The air, the first layer (with $500 \mathrm{~m}$ thickness) and the second layer have the resistivity of $10^{14} \Omega \cdot \mathrm{m}, 200 \Omega \cdot \mathrm{m}$ and $4000 \Omega \cdot \mathrm{m}$, respectively. The conductive semi-torus channel and the ellipsoid have the same resistivity of $10 \Omega \cdot \mathrm{m}$. The center of semi-torus is at the position of $(x=45.9 \mathrm{~km}, y=0 \mathrm{~km}, z=-9.9 \mathrm{~km})$. The diameter of the channel is 1 $\mathrm{km}$. The ellipsoid with a inclination of $45^{\circ}$ is centralized at the position of ( $\left.x=47 \mathrm{~km}, y=0 \mathrm{~km}, z=-11 \mathrm{~km}\right)$. Its $a$-semiaxis, $b$-semiaxis and $c$-semiaxis are $1 \mathrm{~km}, 7 \mathrm{~km}$ and $15 \mathrm{~km}$, respectively.

et al. (1999) and Lin (2010) to study the mesh quality effects on the numerical results of the response of SES. Sarlis et al. (1999) used the model which assumes that the dipole current source lies in the vicinity of a conductive path which terminates below the Earth's surface. Their results indicated that the electric field can be am- plified in the region above the end of the conductive channel. Lin (2010) adopted a similar model to a conductive fault to study the effects of some parameters of the source and fault on the numerical results of electric field. His model results confirmed that the conductive fault or path could explain the selectivity phenomenon 
of SES. His study on the frequency effect of the source indicated that the significant amplified phenomenon of SES would occur mainly at a frequency less than $2 \mathrm{~Hz}$. Therefore, we focused our calculations of the electric field at $1 \mathrm{~Hz}$ in this study.

As the further investigation of meshing effects on the numerical simulation of SES selectivity phe- nomenon, we arranged different elements $\left(n_{4}\right)$ on the arc line $\left(L_{4}=59.92 \mathrm{~km}\right)$ along the top of the conductive path to get a fine or a coarse mesh. Figure $9 \mathrm{a}$ shows a relative fine meshing with $n_{1}=150, L_{1}=160 \mathrm{~km}$, $n_{2}=150, L_{2}=20 \mathrm{~km}, n_{4}=100$, and $L_{4}=59.92 \mathrm{~km}$. Its average $q$ is about 0.701 , with a maximum and a minimum of 0.998 and 0.059 . For a relative coarse meshing in
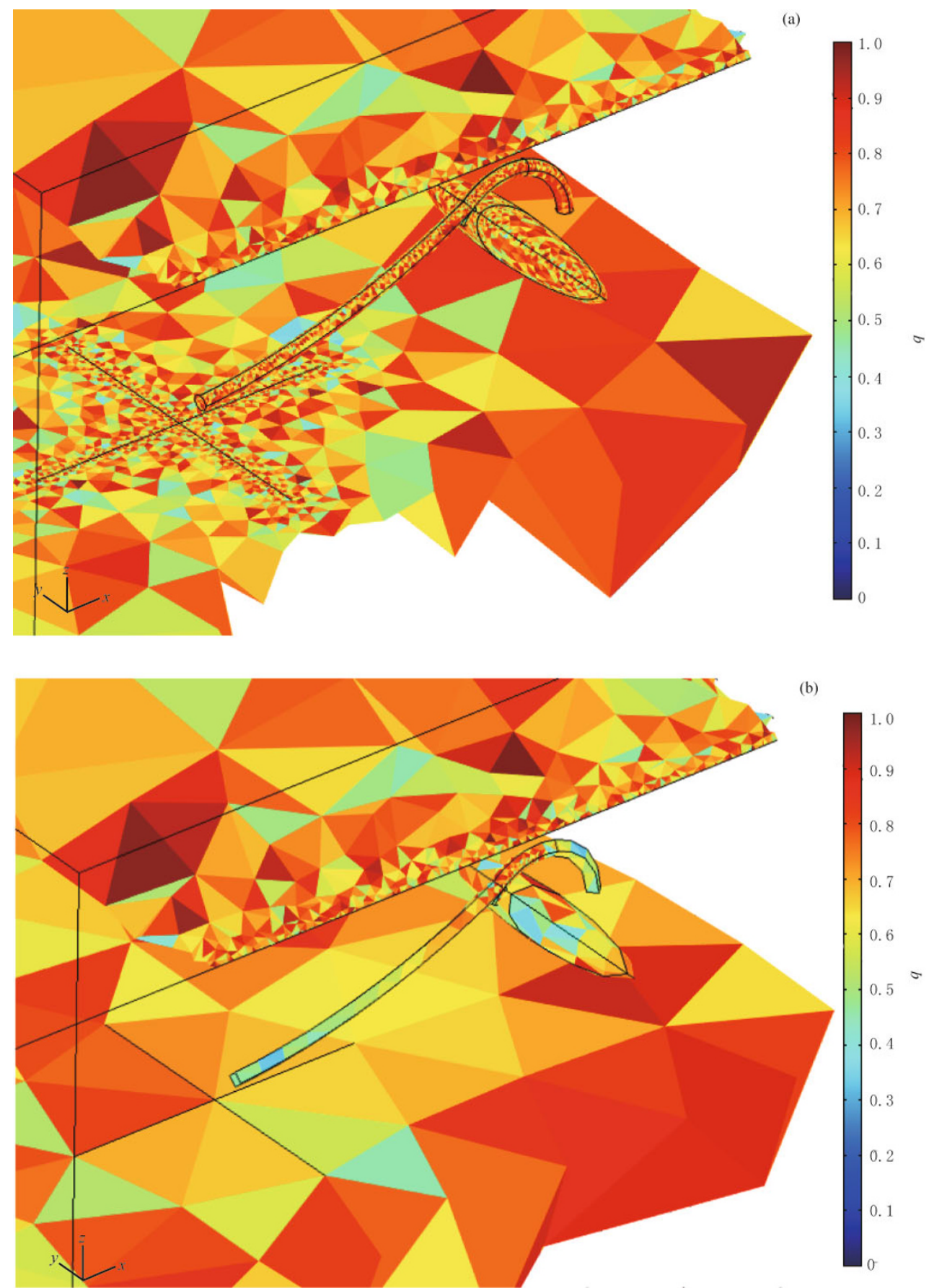

Figure 9 Mesh qualities with different mesh density for the model in Figure 8. (a) A relative fine mesh. (b) A relative coarse mesh. 
Figure $9 \mathrm{~b}$, we let $n_{1}=23, n_{2}=2$, and $n_{4}=35$, and keep $L_{1}, L_{2}$, and $L_{4}$ unchanged. Its average $q$ is about 0.596 , with a maximum and a minimum of 0.989 and 0.014 , respectively.

In Figure 10a, the dotted line is the result from Figure $9 \mathrm{a}$, the dashed line is from Figure $9 \mathrm{~b}$ and the solid line is the theoretical result without conductive

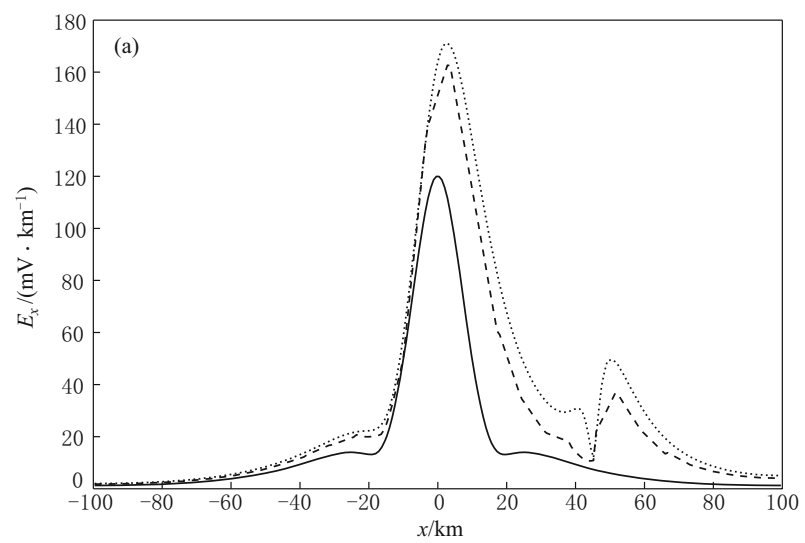

channel and ellipsoid. We can find that on the outcrop (about $x=40-55 \mathrm{~km}$ ) of the channel, the results from a fine meshing condition (dotted line) are larger than that from a coarse meshing condition (dashed line). Their relative errors in the vicinity of the outcrop of the channel could reach $30 \%$ and more (see Figure 10b).

Figure 10 The numerical results of the electric field at $1 \mathrm{~Hz}$ for the model in Figure 8. (a) Dotted line represents the numerical result of a relative fine mesh in Figure 9a, dashed line represents the one of a relative coarse mesh in Figure 9b, and the solid line is the theoretical one without the conductive channel and ellipsoid.

(b) The relative errors between the results of the fine mesh (Figure 9a) and coarse mesh (Figure 9b).

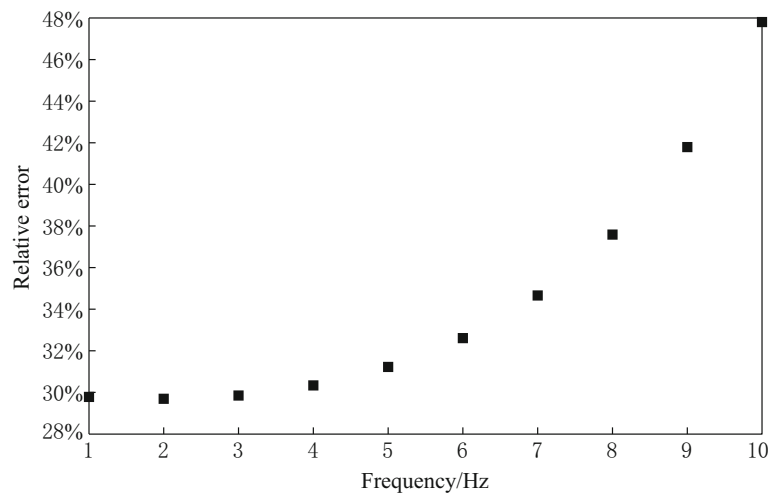

Figure 11 The relative errors at $x=50.8 \mathrm{~km}$ (see text for details) for different frequencies.

In order to investigate the possible frequency effects on the above relative errors, we performed the similar calculations at a frequency varied from $1 \mathrm{~Hz}$ to $10 \mathrm{~Hz}$ using the fine mesh in Figure 9a and the coarse mesh in Figure 9b. Because the point at $x=50.8 \mathrm{~km}$ is the location where a local maximum of the dotted line appears (Figure 10a), for simplicity, Figure 11 shows only the relative errors at $x=50.8 \mathrm{~km}$. It suggested that the relative errors varied non-linearly with frequency. Such effect may lead to a more complicated situation in the numerical simulation of SES selectivity.
Although FEM has been approved an efficient tool for 3D seismo-EM numerical modeling, our study indicated that great care should be taken in explaining SES selectivity based on the numerical results, because the explanation of SES selectivity phenomenon may not be convinced if there is a large relative error due to meshing effect (Figure 10) or other model parameters (Lin, 2010).

Acknowledgements The authors would like to thank Prof. Yongen Cai for some valuable comments on FEM meshing. We thank Mr. Y F Lin for constructive advices of COMSOL software usage. This study was partially supported by the National R \& D Special Fund of Public Welfare Industry (No. 200808069), National Natural Science Foundation of China (Nos. 40974038 and 41025014), and the Joint Research Collaboration Program by the Ministry of Science and Technology of China (No. 2010DFA21570).

\section{References}

Abubakar A, Habashy T M, Druskin V L, Knizhnerman L and Alumbaugh D (2008). 2.5D forward and inverse modeling for interpreting low-frequency electromagnetic measurements. Geophysics 73(4): F165-F177.

Badea E A, Everett M E, Newman G A and Biro O (2001). 
Finite-element analysis of controlled-source electromagnetic induction using Coulomb-gauged potentials. Geophysics 66(3): 786-799.

Bernard P (1992). Plausibility of long-distance electrotelluric precursors to earthquakes. J Geophys Res 97(B12): 17 531-17 546.

Cecot W, Rachowicz W and Demkowicz L (2003). An hp-adaptive finite element method for electromagnetics. Part 3: A three-dimensional infinite element for Maxwell's equations. Int J Numer Meth Engng 57(7): 899-921.

Central Earthquake Working Committee (1969). Proceeding of Summarized Meeting on the Precursors of the $M_{\mathrm{S}} 7.4$ Bohai Earthquake (in Chinese).

COMSOL (2008). COMSOL Multiphysics User's Guide. COMSOL Inc., Berlington, 372pp.

Demkowicz L and Vardapetyan L (1998). Modeling of electromagnetic absorption/scattering problems using hpadaptive finite elements. Comput Meth Appl Mech Engrg 152(1-2): 103-124.

Fraser-Simth A C, Bernardi A, McGill P R, Ladd M E, Helliwell R A and Villard Jr O G (1990). Low-frequency magnetic field measurements near the epicenter of the $M_{\mathrm{S}}$ 7.1 Loma Prieta earthquake. Geophys Res Lett 17: $1456-1468$.

Guo Z Q, Luo X L, Qian S Q, Zhao G F, Guo Z Q, Chen G H, Quan L M and Yan H Z (1999). Electromagnetic emission accompanying mine blasting. Chinese J Geophys 42(6): 834-840 (in Chinese with English abstract).

Hattori K, Serita A, Gotoh K, Yoshino C, Harada M, Isezaki N and Hayakawa M (2004). ULF geomagnetic anormaly associated with 2000 Izu Islands earthquake swarm, Japan. Phys Chem Earth 29: 425-435.

Hayakawa M, Hattori K and Ohta K (2008). Observation of ULF geomagnetic variations and detection of ULF emissions associated with earthquakes: Review. Electr Eng Jpn 162(4): 1 238-1 244.

Hayakawa M, Itoh T, Hattori K and Yumoto K (2000). ULF electromagnetic precursors for an earthquake at Biak, Indonesia on February 17, 1996. Geophys Res Lett 27(10): $1531-1534$.

Hoversten G M and Becker A (1995). EM1DSH with EMMODEL a Motif GUI. University of California at Berkeley.

Huang Q H (2002). One possible generation mechanism of co-seismic electric signals. Proc Jpn Acad B 78(7): 173178.

Huang Q H (2004). The applications of electromagnetics in seismology. Oil Geophys Explor 39(Suppl.): 75-79 (in Chinese with English abstract).

Huang Q H (2005). Controlled analogue experiments on propagation of seismic electromagnetic signals. Chinese Sci Bull 50(17): 1 956-1 961.

Huang Q H and Ikeya M (1998). Seismic electromagnetic signals (SEMS) explained by a simulation experiment using electromagnetic waves. Phys Earth Planet Inter 109(3-4): 107-114.

Huang Q H and Ikeya M (1999). Experimental study on the propagation of seismic electromagnetic signals (SEMS) using a mini-geographic model of the Taiwan Strait. Episodes 22(4): 289-294.

Huang Q H and Lin Y F (2010a). Selecttivity of seismic electric signal (SES) of the $2000 \mathrm{Izu}$ earthquake swarm: A 3D FEM numerical simulation model. Proc Jpn Acad B 86(3): 257-268.

Huang Q H and Lin Y F (2010b). Numerical simulation of selectivity of seismic electric signal and its possible influences. Chinese J Geophys 53(3): 535-543 (in Chinese with English abstract).

Huang Q H and Liu T (2006). Earthquakes and tide response of geoelectric potential field at Niijima station. Chinese $J$ Geophys 49(6): 1 745-1 754 (in Chinese with English abstract).

Jin J, Zunoubi M, Donepudi K C and Chew W C (1999). Frequency-domain and time-domain finite-element solution of Maxwell's equations using special Lanczos decomposition method. Comput Meth Appl Mech Engrg 169: 279-296.

Johnston M (1997). Review of electric and magnetic fields accompanying seismic and volcanic activity. Surv Geophys 18: 441-476.

Key K and Weiss C (2006). Adaptive finite-element modeling using unstructured grids: The 2D magnetotelluric example. Geophysics 71(6): G291-G299.

Kulchitsky A V, Yoshiaki A and Hayakawa M (2004). Numerical analysis on the propagation of ULF/ELF signals in the lithosphere with highly conductive layers. Phys Chem Earth Parts A/B/C 29(4-9): 495-500.

Li Y G and Key K (2007). 2D marine controlled-source electromagnetic modeling. Part 1: An adaptive finiteelement algorithm. Geophysics 72(2): WA51-62.

Lin Y F (2010). Numerical Modeling and Physical Scale Modeling on the Propagtion of Seismic Eletromagnetic Signals. [Master Dissertation]. Peking University, Beijing, 1-61 (in Chinese with English abstract).

Ma S and Liu P C (2006). Modeling of the perfectly matched layer absorbing boundaries and intrinsic attenuation in explicit finite-element methods. Bull Seimol Soc Am 96(5): 1 779-1 794.

Meng Z and Dosso H W (1990). An analogue model study of EM induction in the Japan-Korea-China region. Phys Earth Planet Inter 62(3-4): 246-256.

Mitsuhata Y and Uchida T (2004). 3D magnetotelluric modeling using the $T-\Omega$ finite-element method. Geophysics 69(1): 108-119.

Mulder W A (2006). A multigrid solver for 3D electromagnetic diffusion. Geophysical Prospecting 54(5): 633-649.

Nagao T, Enomoto Y, Fujinawa Y, Hata M, Hayakawa M, 
Huang Q H, Izutsu J, Kushida Y, Maeda K, Oike K, Uyeda S and Yoshino T (2002). Electromagnetic anomalies associated with 1995 Kobe earthquake. J Geodyn 33(4-5): 401-411.

Nam M J, Kim H J, Song Y, Lee T J, Son J S and Suh J H (2007). 3D magnetotelluric modelling including surface opography. Geophys Prop 55(2): 277-287.

Ndlec J C (1980). Mixed finite elements in $\mathbf{R}^{3}$. Numer Math 35: $315-341$.

Ndlec J C (1986). A new family of mixed finite elements in $\mathbf{R}^{3}$. Numer Math 50: 57-81.

Qian J, Chen Y and Jin A (1985). Geoelectric Resistivity Method and its Application in Earthquake Prediction. Seismological Press, Beijing, 274pp (in Chinese).

Rachowicz W and Demkowicz L (2000). An hp-adaptive finite element method for electromagnetics. Part 1: Data structure and constrained approximation. Comput Methods App M 187(1-2): 307-335.

Rachowicz W and Demkowicz L (2002). An hp-adaptive finite element method for electromagnetics. Part II: A 3D implementation. Int J Numer Meth Eng 53(1): 147-180.

Ren Z Y, Tang J T and Liu C S (2008). Edge finite-element modeling using unstructured meshes: The 3-D MT example. In: Insitute of Geology, China Earthquake Administration and Geo-Electromagnetic Committee, Chinese Geophysical Society eds. IAGA WG 1.2 on Electromagnetic Induction in the Earth, Extended Abstract 19th Workshop. Beijing, October 23-29, 2008, S5_E06, 520-525.

Sarakorn W, Siripunvaraporn W and Gary E (2008). Threedimensional magnetotelluric modeling using finite element method: Tetrahedral and hexahedral elements, comparision to finite difference method. In: Insitute of Geology, China Earthquake Administration and GeoElectromagnetic Committee, Chinese Geophysical Society eds. IAGA WG 1.2 on Electromagnetic Induction in the Earth, Short Abstract 19th workshop. Beijing, October 23-29, 2008, S5_S38, 719.

Sarlis N, Lazaridou M, Kapiris P and Varotsos P (1999). Numerical model of the selectivity effect and the $\Delta V / L$ criterion. Geophys Res Lett 26(21): 3 245-3 248.

Sobolev G A (1975). Application of electric method in the tentative short-term forecast of Kamchatka earthquake. Pure Appl Geophys 113: 229-235.

Sugeng F (1998). Modeling the 3D TDEM response us- ing the 3D full-domain finite-element method based on the hexahedral edge-element technique. Exploration Geophysics 29(4): 615-619.

Tang J T and Wang F Y (2008). 2.5-D direct current resistivity forward modeling with unstructured mesh. In: Insitute of Geology, China Earthquake Administration and Geo-Electromagnetic Committee, Chinese Geophysical Society eds. IAGA WG 1.2 on Electromagnetic Induction in the Earth, Extended Abstract 19th Workshop. S5_E04 Beijing, October 23-29, 2008, S5_E04, 509-513.

Vallianatos F and Tzanis A (1998). Electric current generation associated with the deformation rate of a solid: Presseismic and coseismic signals. Phys Chem Earth 23(9-10): 993-938.

Varotsos P (2005). The Physics of Seismic Electric Signals. Terra Scientific Publishing Company, Tokyo, 1-388.

Varotsos P and Alexopoulos K (1984a). Physical properties of the variations of the electric field of the earth preceding earthquakes (I). Tectonophysics 110: 73-98.

Varotsos P and Alexopoulos K (1984b). Physical properties of the variations of the electric field of the earth preceding earthquakes (II): Determination of epicenter and magnitude. Tectonophysics 110: 99-125.

Varotsos P and Lazaridou M (1991). Latest aspects of earthquake prediction in Greece based on seismic electric signals. Tectonophysics 188(3-4): 321-347.

Varotsos P, Sarlis N, Lazaridou M and Kapiris P (1998). Transmission of stress induced electric signals in dielectric media. J Appl Phys 83(1): 60-70.

Wei J B, Zhang G J and Liu Q H (2007). Recursive algorith$\mathrm{m}$ and refined calculation of the dyadic Green's functions in layered uniaxial anisotropy media. Science in China 37(6): 836-850.

Xu S (1994). FEM in Geophysics. Science Press, Beijing, 308pp (in Chinese).

Yoshimura R and Oshiman N (2002). Edge-based finite element approach to the simulation of geoelectromagnetic induction in a 3-D sphere. Geophys Res Lett 29(3): 1039.

Zhao Y and Qian F (1978). Electrical resistivity anomaly observed in and around the epicentral area prior to the Tangshan M7.8 earthquake of 1976. Acta Geophysica Sinica 21: 181-190 (in Chinese with English abstract).

Zhdanov M S (2009). Geophysical Electromagnetic Theory and Methods. Elsevier, Oxford, 1-845. 